\title{
el suelo estabilizado, material de construcreion
}

la compactación de suclos

1. NADAL, ingeniero de caminos

\section{Objetro de la compactación}

Mediante la compactación de un suelo se pretende lograr: que su volumen permanezca prácticamente constante; que su resistencia se encuentre siempre por encima de un mínimo admisible, y que las deformaciones producidas por las cargas a que esté sometido se mantengan dentro de límites tolerables.

De todos los sistemas posibles para lograr estas propiedades, la compactación se basa exclusivamente en reducir el volumen de huecos.

\section{Densidad del suelo}

La densidad de un suelo depende, fundamentalmente, de:

a) Densidad real de sus partículas sólidas.

b) Volumen de huecos.

c) Agua contenida en los huecos.

De estos tres parámetros, el primero depende únicamente de la naturaleza del suelo, de tal suerte que, al considerar un suelo determinado, la densidad real de sus partículas sólidas es, a efectos de la compactación, una constante característica y, por lo tanto, la densidad varía únicamente con el volumen de huecos y el agua que contienen. 


\section{Densidad en seco}

A efectos de reproducibilidad de los ensayos, y para poder comparar sus resultados, es muy frecuente-en la técnica que nos ocupa-desecar las probetas a peso constante, antes de determinar su densidad, por lo que ésta, en cada suelo, varía únicamente con el volumen de huecos, lo que permite asimilar el grado de compactación al valor de la densidad en seco.

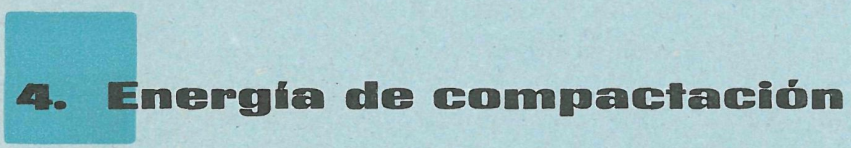

Para reducir el volumen de huecos de un suelo es preciso hacer que sus partículas sólidas se muevan, de tal suerte que vayan rellenando los huecos existentes.

Para lograr estos desplazamientos relativos es necesario suministrar energía al sistema; ahora bien, de esta energía sólo una parte se invierte en reordenar convenientemente las partículas, existiendo, por lo tanto, un cierto rendimiento-en general, muy bajo-que depende de muy diversos factores.

Se han hecho muchas y muy interesantes investigaciones encaminadas a destacar los factores determinantes $y$, en definitiva, a aumentar los rendimientos, pero el problema es complejo por las dificultades que entrañan siempre estos trabajos de investigación y porque, en última instancia, es la industria de la maquinaria auxiliar la que debe estar en condiciones de dar forma industrial a los resultados que los laboratorios y las obras van obteniendo.

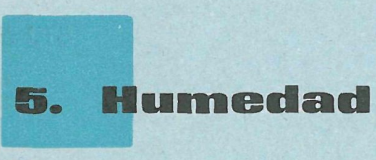

Evidentemente, uno de los factores que determinan el rendimiento es la resistencia que las partículas oponen a sus mutuos desplazamientos.

En definitiva, es un problema mecánico de enlaces interiores; y como tal, cabe tratarlo por el sistema tradicional de la lubricación. El agua es el único lubricante que las condiciones económicas de las obras han permitido utilizar hasta hoy, tanto más cuanto que este elemento se encuentra siempre en el suelo y en muchos casos no es necesario ni siquiera añadirlo como nueva aportación.

La dificultad estriba en poder determinar «a priori» cuál es la proporción de agua conveniente para que la película adherida a los sólidos tenga propiedades lubricantes, ya que si el agua se añade en exceso, ésta y el aire ocluído actúan de dispersantes, y si, por el contrario, no se alcanza la cantidad necesaria, el trabajo se hace penoso y baja el rendimiento.

\section{Humedad óptima}

Se ha comprobado-y siempre nos referimos a un mismo suelo-que la densidad depende, dentro de ciertos límites, de la energía de compactación y del contenido de humedad durante la operación de compactado. Esto permite fijar, de antemano, una energía de compactación constante e ir variando, sucesivamente, el contenido de agua, con lo que se obtienen curvas como la que se incluye en la figura 1, que presenta un máximo. Este máximo determina la llamada humedad óptima, que lo es respecto de una determinada energía, pero que, en general, varía al variar ésta. 

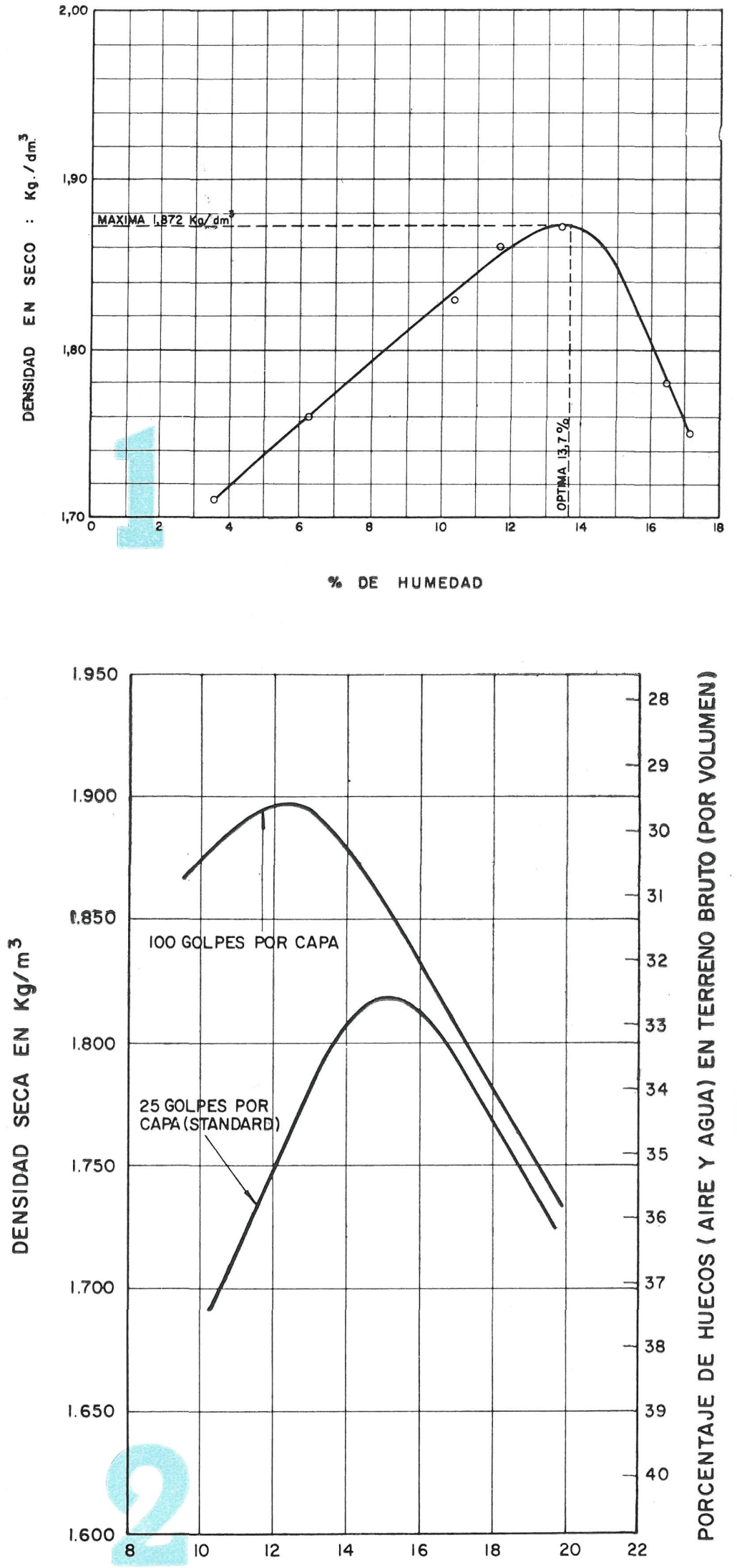

PORCENTAJE DE HUMEDAD CONTENIDA relación humedacd

diensidiand

factor morimeal

A efectos de las aplicaciones prácticas, la energía que se toma, constante para todos los ensayos, se ha fijado de manera que los resultados sean representativos y correspondan, en lo posible, a las que realmente hayan de obtenerse en obra, con los medios normales hoy al alcance de los constructores, pero que se debe señalar que, tanto los ensayos en sí como los valores de las constantes a que nos referimos. se han establecido fundamentalmente para aplicaciones del suelo estabilizado en vías đe comunicación-carreteras, aeropuertos. etcétera--, y que, si bien pueden por extensión aplicarse en ciertos casos a rellenos sobre los que luego hubiera de edificarse. su aplicación a los tapiales y, en general, a los problemas del edificio, requeriría una cuidadosa revisión de los métodos.

\section{․ Variaciones de la emergía dé commpactación}

Como ya hemos apuntado, para cada suelo, el aumento de energía aplicada incrementa la densidad en seco v disminuye el valor de la humedad óptima.

En los laboratorios, la energía se aplica, normalmente, dejando caer una maza, desde cierta altura, sobre la muestra una vez colocada en moldes y repitiendo esta operación un determinado número de veces. Los resultados que se obtienen sobre un mismo suelo, cuando se aumenta el número de golpes, pueden verse en la figura 2. 

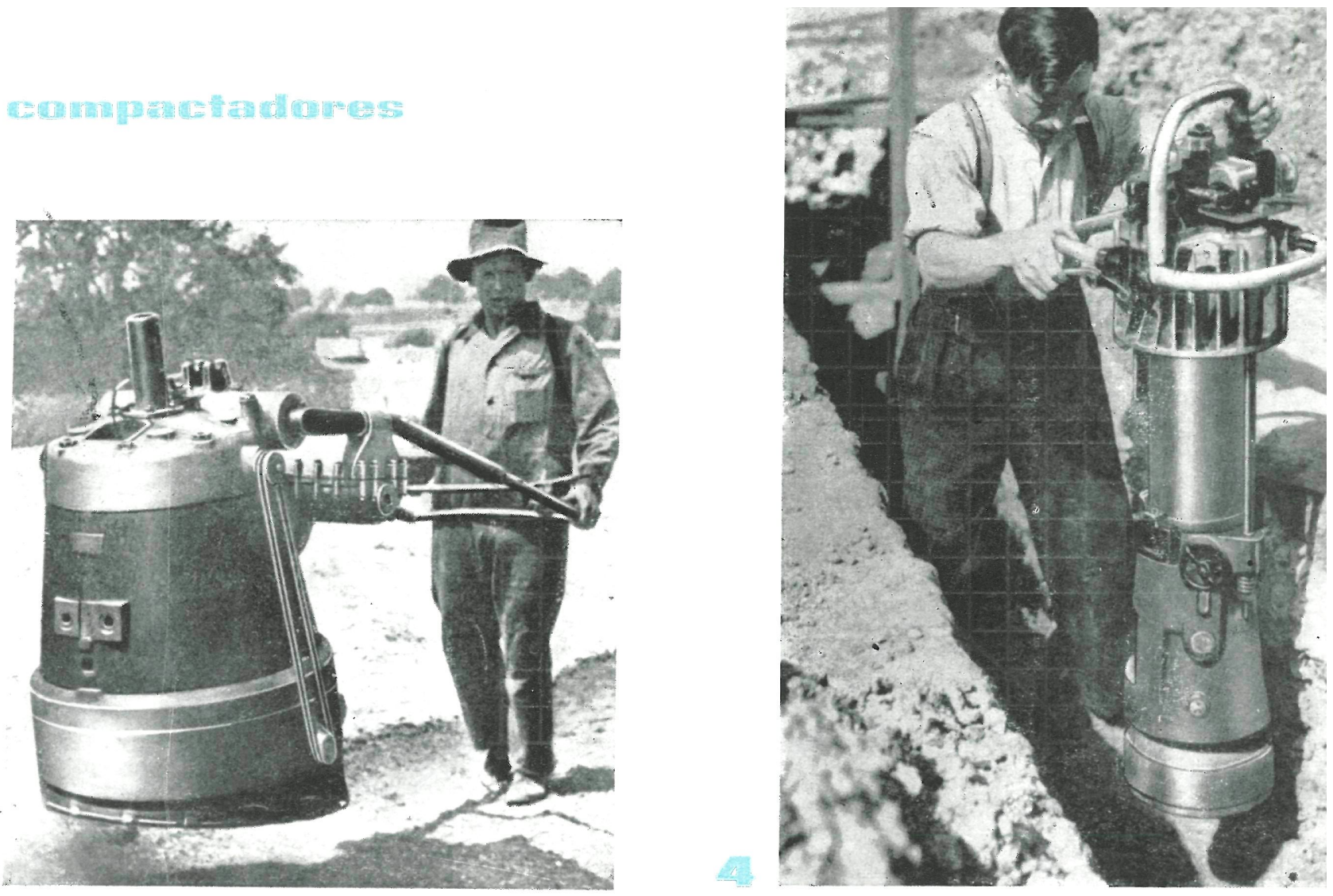

\section{Métodos de compactación}

La energía que un suelo absorbe para su compactación depende de la forma en que se aplique esta energía. Dicho de otro modo: para cada suelo hay unos métodos de compactación más apropiados que otros.

La dificultad práctica estriba en que no siempre los métodos de compactación apropiados desde el punto de vista que ahora nos ocupa. lo son si se consideran desde el ángulo de las posibilidades del constructor.

La industria, en general y, concretamente, la de productos químicos, aglomera o compacta las sustancias pulverulentas por presión estática, sistema que en construcción no puede aplicarse más que para la fabricación de pequeños elementos, tales como bloques de edificación y otras piezas prefabricadas.

La compactación por pisones manejados a brazo, tan característica de las pequeñas obras. tiene en la construcción actual dos expresiones mecánicas, que son las máquinas de pisones utilizadas en ciertos trabajos de estabilización en carreteras y las llamadas ranas (figs. 3 y 4), pero es en el laboratorio donde el apisonado vertical tiene aún su mayor aplicación. 

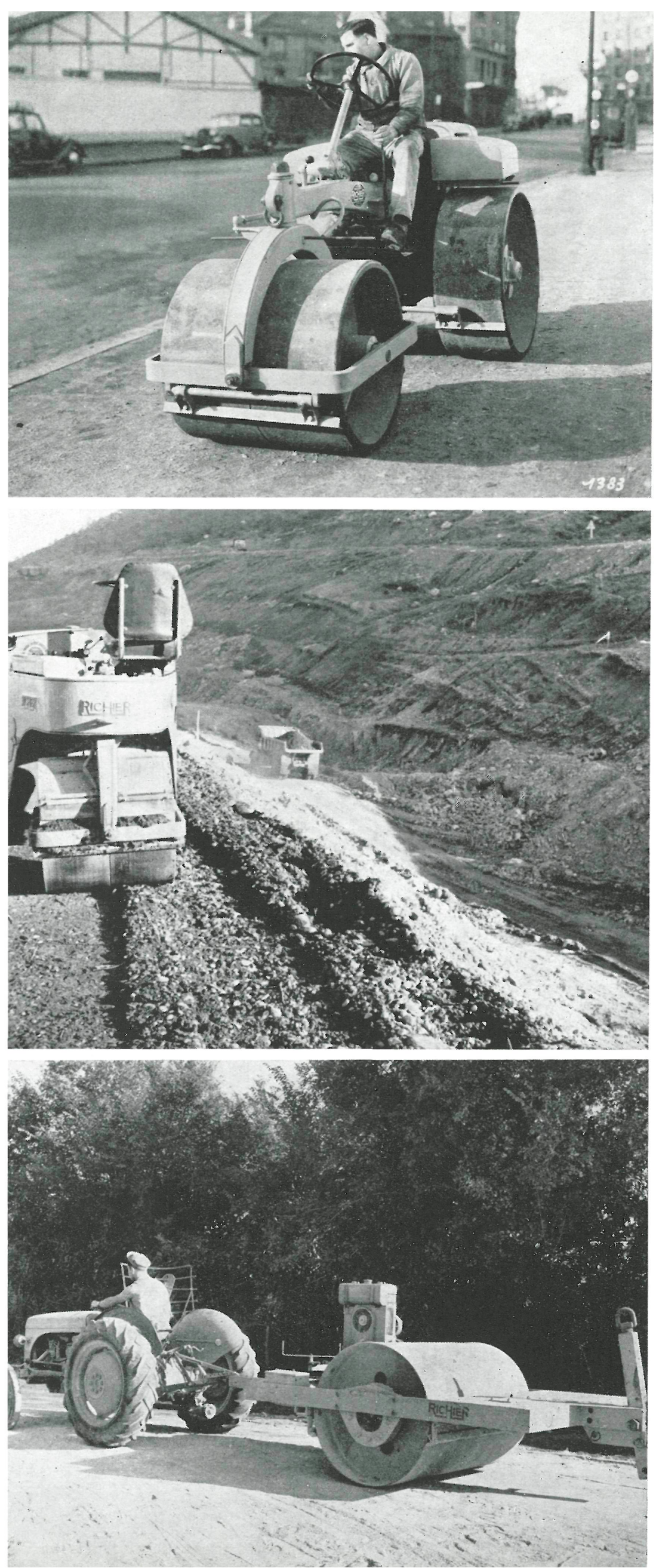



La vibración de suelos se practica mediante diversos ingenios, que van desde el vibrador de patín de sólo unos kilos de peso (fig. 5), a la viga vibrante, pasando por los rodillos apisonadores - vibradores (figuras 6 a 9).

Los rodillos de pie de cabra son, junto con las apisonadoras de neumáticos, aportaciones relativamente recientes de la industria de maquinaria (figs. 10 a 13), y su utilización se ha extendido grandemente en los últimos años. Por último, aún subsisten las apisonadoras de rueda lisa o corrugada, según distintos tipos y modelos, cuyos pesos van de una tonelada a más de veinte (figs. 14 a 17). 


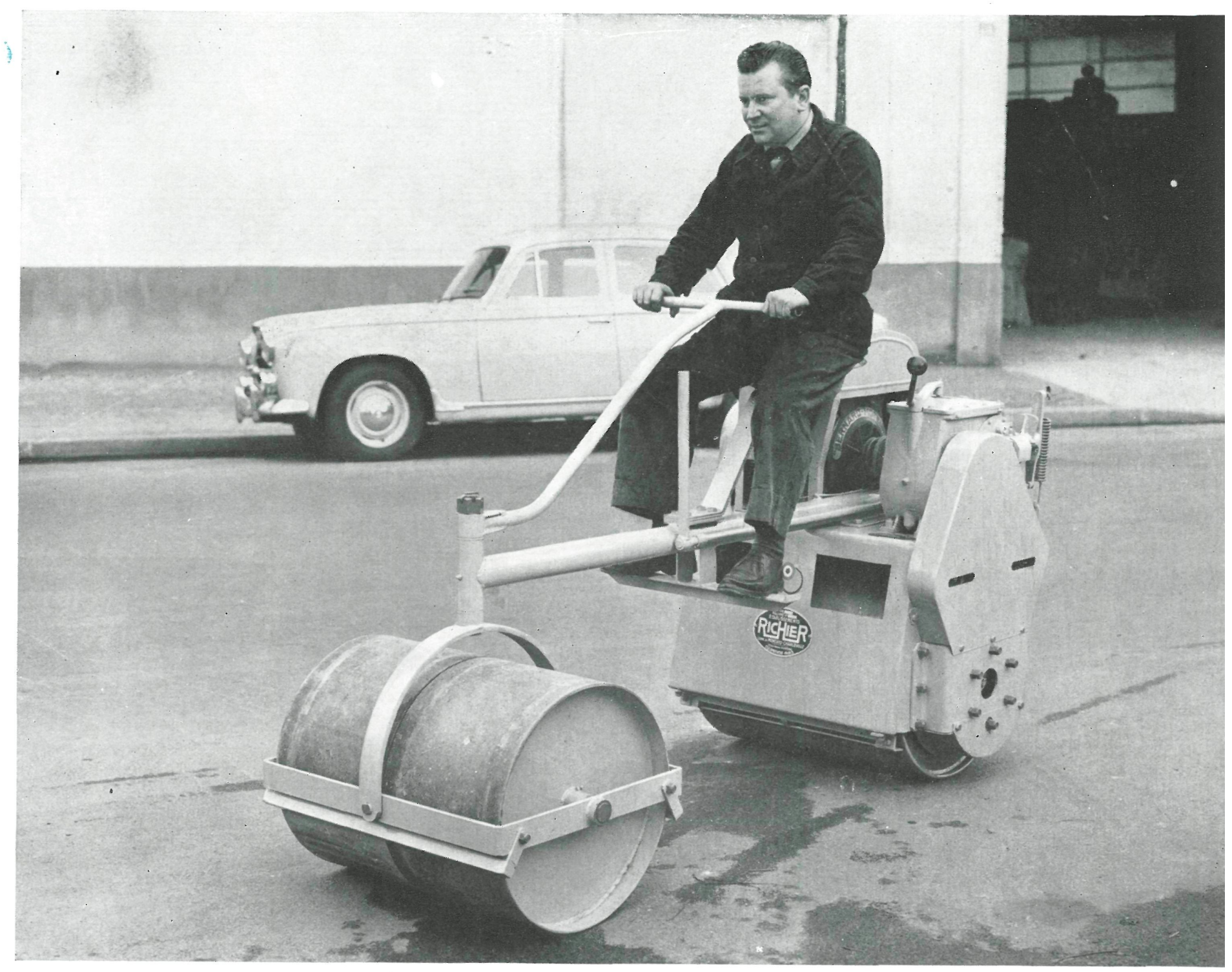

9

\subsection{SUELOS GRANULARES}

El moldeo de piezas con conjuntos granulares está, en líneas generales, bien resuelto industrialmente, entre otras razones porque es, en esencia, el problema que plantea la puesta en obra de los morteros y hormigones, cuyas técnicas han progresado mucho estos últimos años y, sobre todo, están en constante evolución, especialmente, por el interés que ello tiene para la industria de prefabricados. La dificultad que puede ofrecer su aplicación a los suelos propiamente dichos es esencialmente económica. 


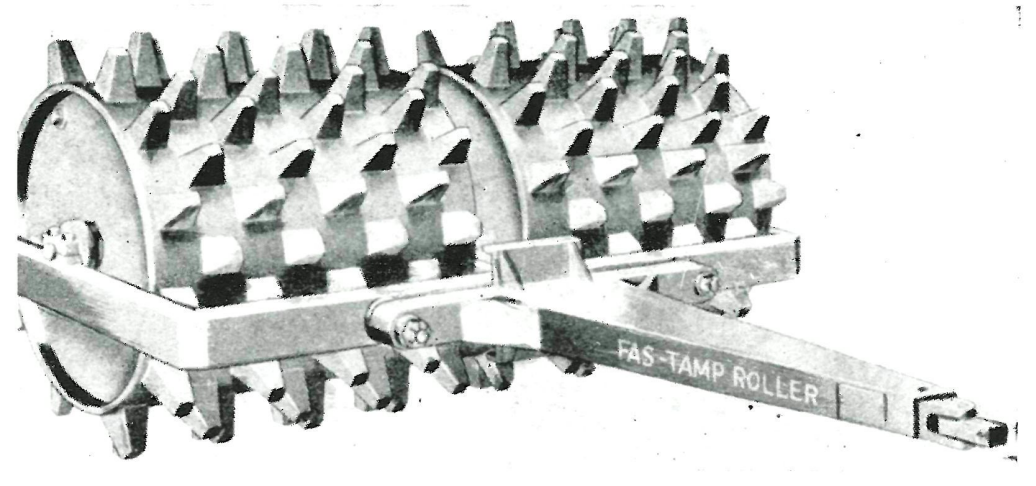

Tili

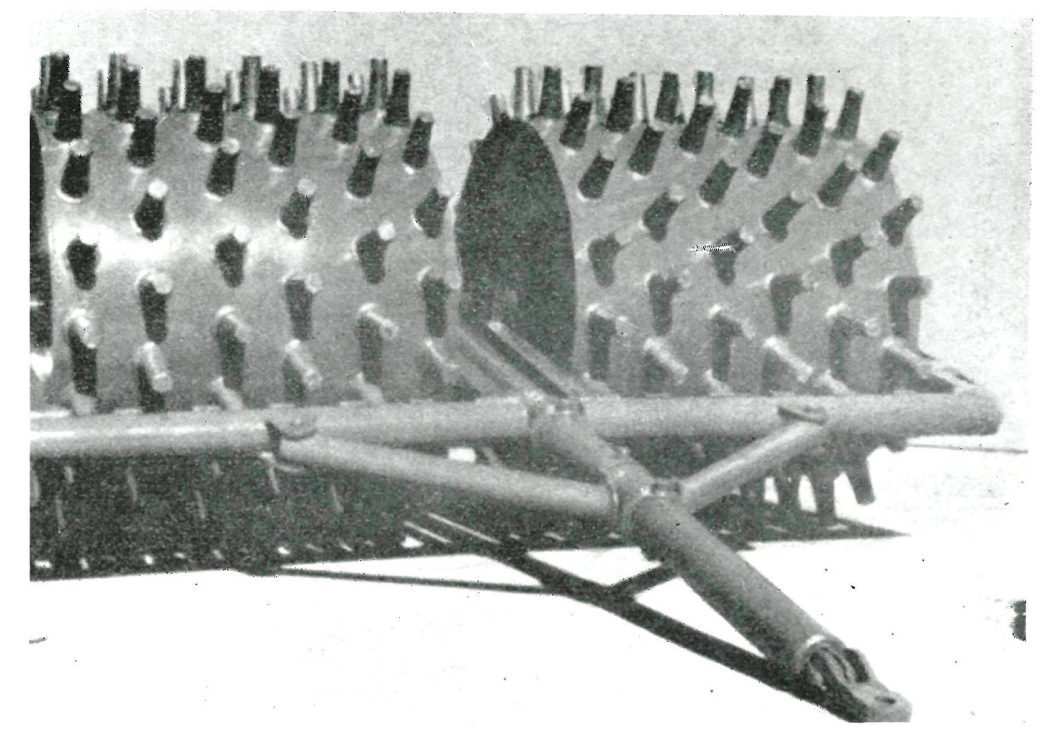

需盾

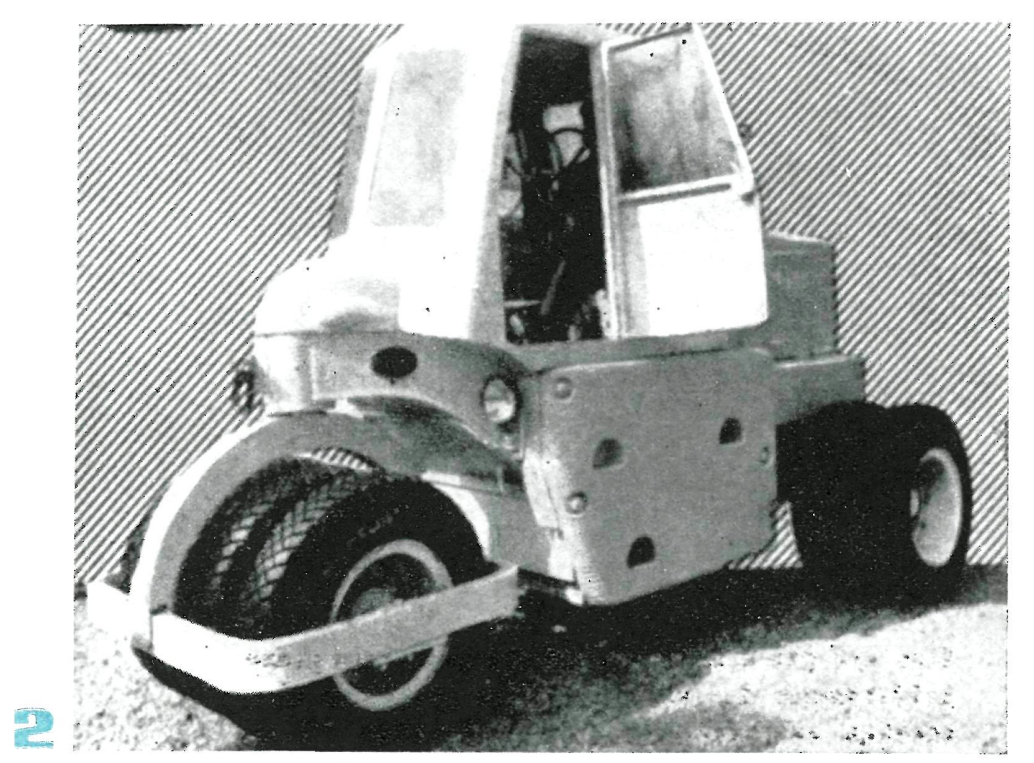

\subsection{SUELOS COHESIVOS}

La compactación de suelos cohesivos para su utilización en edificación sin posterior tratamiento estabilizador no se ha realizado más que con motivo de los tapiales y de los adobes, y sobre ellos no ha recaído aún un estudio sistemático sobre bases científicas.

La estabilización de un suelo natural por simple acción mecánica, ya hemos visto, en otros lugares, que no es casi nunca posible, o, mejor dicho, que no es posible más que en determinadas circunstancias especiales. Su utilización está, además, muy ligada a consideraciones económicas, ya que lo verdaderamente útil y barato sería utilizar, como se ha dicho muchas veces, los productos de las excavaciones para explanaciones y cimentaciones como material gratis a pie de obra con el que constituir los muros. Esto se da muy raramente.

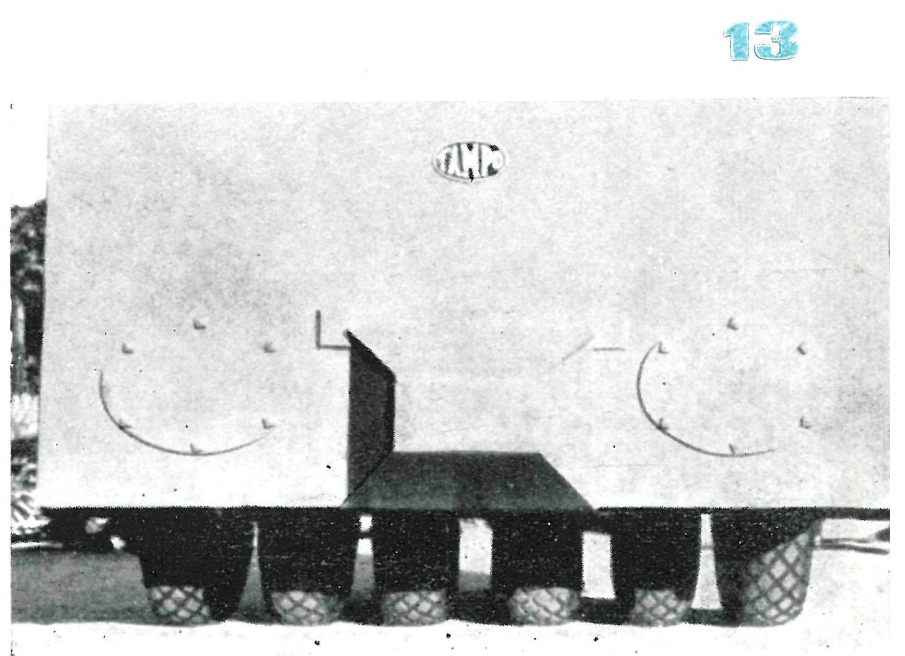



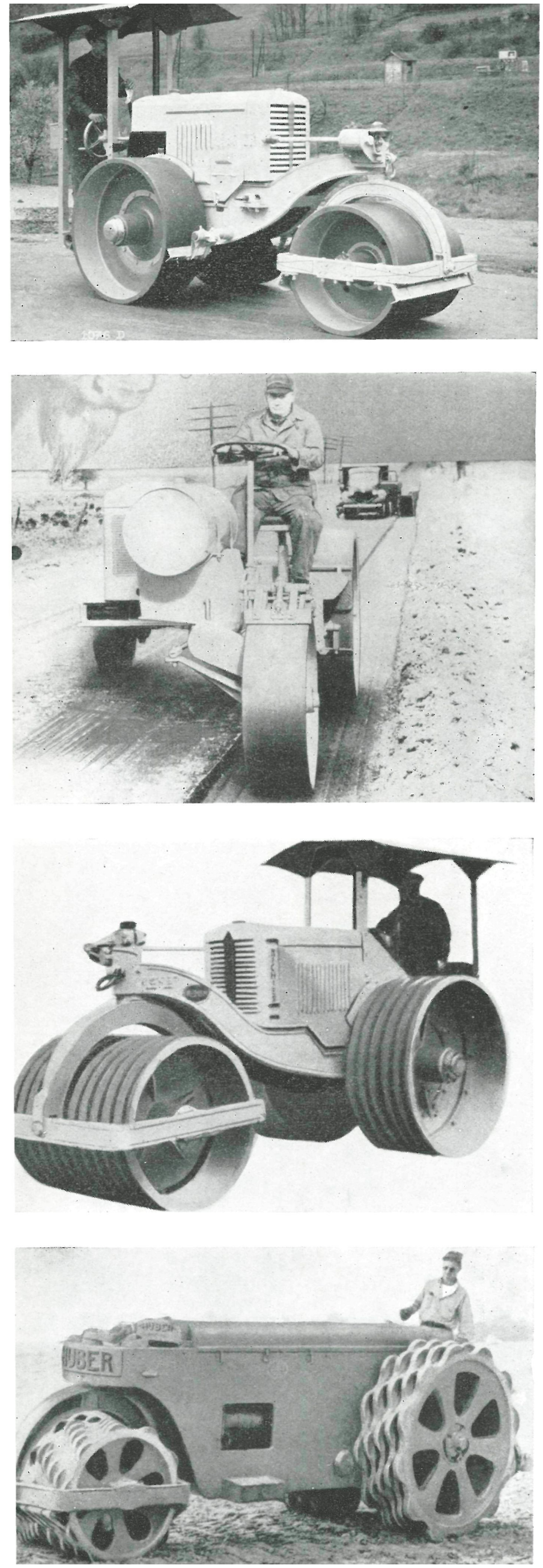

Es poco frecuente que, precisamente, la tierra que se extraiga cumpla con las condiciones de composición, granulometría y naturaleza, que serían precisas e, incluso, comprobarlo puede ofrecer ciertas dificultades de orden práctico difíciles de superar sin la colaboración de un laboratorio, lo que puede ser grave inconveniente si la obra es muy pequeña.

\subsection{COMPACTACION DE TERRAPLENES}

Los terraplenes se compactan para poder construir inmediatamente encima sin peligro de asientos; reducir la tendencia del suelo a aumentar su grado de humedad, con la consiguiente pérdida de capacidad resistente y cambios de volumen; y, finalmente, los terraplenes se compactan también para evitar los peligros de corrimientos y aumentar la capacidad resistente.

Las máquinas que se utilizan a tal fin, son, como ya se ha dicho, apisonadoras de diversos tipos, rodillos de neumáticos y vibradores. El empleo de unos u otros depende de la naturaleza del suelo y del grado de humedad, no existiendo reglas fijas que permitan determinar, «a priori», con exactitud, el tipo de máquina más apropiada y su posible rendimiento, por lo cual es recomendable determinarlo mediante pruebas previas en la propia obra. Es también muy corriente que los mejores resultados se logren combinando varias máquinas distintas, por lo que no es raro ver en una obra trabajando en serie varias de ellas.

A falta de otros datos más concretos, y como orientación para la elección de maquinaria y predeterminación de su posible rendimiento, se consideran especialmente útiles los ensayos realizados por diversos Centros de investiga- 